\title{
Estado de la investigación clínica en Colombia
}

\section{State of the clinical research in Colombia}

\author{
Dora Inés Molina de Salazar, Miguel Álvarez-Mejía • Manizales (Colombia)
}

\section{Introducción}

La investigación médica es esencial para hacer frente a los retos del futuro, ya que su resultado no sólo produce nuevos conocimientos, ciencia e innovación, sino que también puede ser traducido en mejores formas de prevenir y tratar las enfermedades (1). Es por ello, que diversos países se han sumergido en este campo investigativo y por ende les ha permitido un mejor desarrollo en salud, tecnología y políticas macro económicas con un aumento de inversión en capital extranjero; para junio de 2017 a nivel mundial existían 289227 estudios clínicos, según Clinical Trails.gov, los cuales presentan una mayor densidad en países como Estados Unidos y los pertenecientes al continente europeo, con 116237 y 81769 respectivamente (2); en cuanto a los países del continente asiático, principalmente en la región del sureste, han tenido un gran desarrollo en los últimos años con 31071 para junio del año anterior $(1,2)$; respecto a Colombia, La investigación clínica data de mas de 30 años, sin embargo, es a partir de la década de los 90 en donde se alcanzan los estándares necesarios para poder competir con estudios internacionales, esto ocurre gracias a la aplicación desde el INVIMA de las resoluciones 8430,3823 y 2378 (3).

En 2001 la Organización Panamericana de la Salud estableció un grupo de trabajo (Conferencia Internacional de Armonización de Buenas Prácticas Clínicas 1993), para dar a conocer las buenas practicas clínicas de investigación BPC (GCP Good Clinical Practice) y como resultado de ello se produjo un documento de BPC de las Américas en 2005, el cual es aplicable a los estudios clínicos de intervención farmacológica.

El desarrollo de un estudio clínico, involucra cierto grado de complejidad tanto en su estructura como implementación, ya que requiere de un equipo multidisciplinario a la hora de llevar a cabo este proceso; exige la intervención coordinada de patrocinadores CRO's, investigadores principales y secundarios, monitores, coordinadores, químicos farmacéuticos, regentes de farmacia, comités de ética, entidades regulatorias, empresas de transporte, aduanas y comercio exterior, servicios de mensajería especializada, entre otros. Aunado a esto, la participación voluntaria de sujetos en investigación posterior a la firma del consentimiento informado (4).

Está presupuestado, según la asociación Colombiana de Investigación Clínica (ACIC), que para poder funcionar un centro de investigación clínica en Colombia se requiere de una inversión aproximada para el día de hoy de $\$ 1100000000 \mathrm{COP}$, con un costo mensual de operación de $\$ 100000000$ - $800000000 \mathrm{COP}$, se requiere un personal mínimo de base para funcionamiento, que debe rondar entre 5-7 personas, con un nivel educativo muy alto: $20 \%$ con postgrado, $60 \%$ con titulo universitario, $10 \%$ con nivel técnico, $10 \%$ con bachillerato (5).

Respecto a los tiempos para iniciar un estudio después de ser aprobado por el INVIMA para la mayoría está por encima de lo esperado ver (Figura 1).

\section{La investigación clínica patrocinada en el mundo}

La investigación clínica patrocinada tiende a crecer con un ritmo superior al de la economía mundial. El valor del mercado oscila entre 15 y 90 millardos (miles de millones de dólares), de acuerdo con análisis realizados por Research and Markets será de US 64
Dra. Dora Inés Molina de Salazar: Profesora Titular, Facultad de Ciencias para la Salud Universidad de Caldas. Fellow of the American College of Physicians (FACP). Fellow LatinAmerican Society of Hypertension (LASH). Director Clinical Research, Asociación IPS Médicos Internistas de Caldas; Dr. Miguel Álvarez-Mejía: MD Universidad de Caldas. Manizales (Colombia).

Correspondencia: Dra. Dora Inés Molina de Salazar. Manizales (Colombia).

E-mail: doraines56@gmail.com

Recibido: 6/XI/2018 Aceptado: 13/XI/2018 


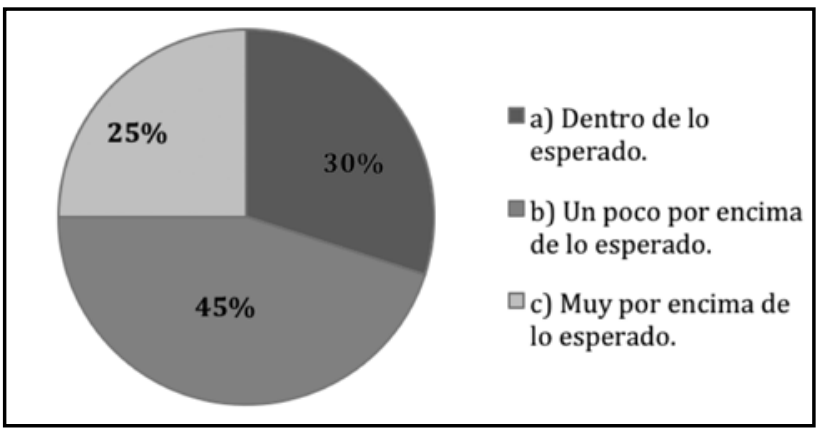

Figura 1. Tiempos para inicio de estudios después de aprobación Invima. Tomado y adaptado de (5).

millardos en el 2020, con un crecimiento anual ponderado del $5.5 \%$.

La cantidad de investigación clínica patrocinada va en aumento a nivel mundial, según welocalize.com, éstos han aumentado de 35835 - 234955 entre los años 2006 y 2016, concomitantemente, el número de países hacedores de estos estudios aumentó de forma paralela, pasando de 92 - 140 durante este intervalo de tiempo.

Según Research and Markets los países emergentes son responsables de $15.7 \%$ de los estudios clínicos patrocinados y está proyectado que para el 2020 éstos tendrán la responsabilidad de $25.2 \%$ de todos los estudios (6).

Los principales hechos que permitieron el desarrollo de más estudios clínicos en América del Sur en los últimos 20 años se explican por:

1. Estabilización política con predominio de la democracia.

2. Desarrollo económico importante que permitió inversión de capital extranjero.

3. Cooperación en el área científica internacional, contando con centros académicos, personal capacitado en investigación clínica (7).

Esto ha permitido que las compañías de investigación y desarrollo tecnológico otorguen a Colombia la posibilidad de realizar estudios clínicos principalmente en fases II, III, IV. (7).

\section{Desempeño de la investigación clínica en Colombia}

La investigación clínica en Colombia en la última década ha mostrado un desarrollo interesante y se ha convertido en un competidor importante en la región. Según Clinical Trials.gov -la base de datos de estudios clínicos más grande del mundo administrada por el "Instituto Nacional de Salud" (National Institute of Health)- NIH por sus cifras en ingles-. En Colombia para el año 2017, se encuentran 1185 estudios registrados respecto al 2016 donde se tenían 927 para un aumento de $1.27 \%(2,7)$. Estas son cifras alentadoras para los investigadores en Colombia, y para los nuevos que quieren entrar en esta área del conocimiento. Sin embargo, aun cuenta con un rezago regional, ya que se encuentra ubicada en el cuarto puesto a nivel de Latinoamérica después de Brasil, Argentina y Chile, (5).
Tabla 1. Estudios clínicos radicados en el INVIMA 2008-2017.

\begin{tabular}{|l|c|c|c|}
\hline \multicolumn{4}{|c|}{ Comparación por quinquenios y totales } \\
\hline Quinquenio & $\begin{array}{c}\text { EC } \\
\text { radicados }\end{array}$ & $\begin{array}{c}\text { EC } \\
\text { aprobados }\end{array}$ & $\begin{array}{c}\text { EC } \\
\text { no aprobados }\end{array}$ \\
\hline $2008-2012$ & 454 & 411 & 43 \\
\hline $2013-2017$ & 425 & 368 & 57 \\
\hline Variación & $-6 \%$ & $-10 \%$ & $33 \%$ \\
\hline Totales & 879 & 779 & 100 \\
\hline Promedio año & 88 & 78 & 10 \\
\hline \multicolumn{2}{|c|}{ Tomado y adaptado de (4). }
\end{tabular}

Colombia debe hacer un esfuerzo para mejorar sus tiempos regulatorios entre el INVIMA, comités de ética, centros de investigación, y CRO, y así, se volvería más atractivo y competitivo para nuevos ensayos clínicos.

Actualmente en Colombia, según el INVIMA, existen 120 instituciones certificadas con Buenas Practicas Clínicas, 70 comités de ética, 19 instituciones con certificación cancelada (8).

Sin embargo, respecto a la información disponible en la pagina del INVIMA, en Colombia entre 2008 y 2017 se radicaron en la agencia 879 estudios clínicos, con un porcentaje de aprobación del $89 \%$, al distribuir esta información en quinquenios, entre 2008-2012 hubo 6\% más ensayos clínicos radicados y $10 \%$ más ensayos clínicos aprobados que el quinquenio 2013-2017; en este ultimo, la cantidad de ensayos clínicos radicados y aprobados permaneció por debajo de la media (4) (Tabla 1).

En cuanto a la densidad de centros de investigación, Colombia, cuenta con un índice de 2.5 centros por cada millón de habitantes, mientras que, países como Alemania, Francia, o Reino Unido tienen un índice de 50 centros por cada millón de habitantes, en Estados Unidos, el índice aumenta a 120 por millón de habitantes; En Colombia la densidad de participantes por millón de habitantes es de tres, Perú y Brasil cuatro por millón, México: seis por millón, Chile: once por millón (7). Estos indicadores muestran la hazaña a la que se enfrentan los investigadores en Colombia para poder competir incluso de forma internacional.

Respecto a las metas de reclutamiento a las que se ven sometidos los Centros de Investigación, según ACIC en el año $2017,58 \%$ cumplieron las metas, $37 \%$ no cumplieron las metas, y 5\% están en curso de cumplirlas; los estudios de cáncer usualmente cumplen la meta entre 70 y 100\%, reumatología/nefrología $50 \%$, cardiología y neumología 40-50\%. Las principales razones para no alcanzar dichos objetivos son los tiempos cortos para reclutar, el hecho de un inicio paralelo a Colombia de reclutamiento en otros países, la meta global de los estudios es alcanzada antes de que Colombia termine de reclutar, Colombia entra a reclutar como centro de rescate, hay tiempos largos para el inicio, y existe demora en entrega de insumos y medicamentos (5). 
De los 1164 protocolos de investigación, las fases de estudios clínicos más frecuentes fueron:

- Fase III, 47\%

- Fase II $15 \%$.

- Fase IV 7\%.

- Observacional 4\%.

- Acceso expandido $2 \%$.

- Otros $1 \%$.

- Fase I $1 \%$.

- Post-mercadeo 0.2\%.

Para el año 2013 las instituciones mayormente involucradas en las diferentes fases de investigación clínica (I a IV), fueron la Universidad Javeriana, la Fundación Cardioinfantil, el Instituto de Cardiología, el Instituto Nacional de Cancerología y la Universidad de Antioquia (9).

En una investigación de carácter descriptiva y cualitativa de los 1164 estudios clínicos registrados en INVIMA desde el año 1999 - 2009, los temas más estudiados fueron:

- Fármacos (88.1\%).

- Estudios de intervención (71\%).

- Medicamentos para el sistema cardiovascular (13\%).

- Casa patrocinadora (22.3\%).

- Estudios fase III (47\%).

- Tamaño de muestra entre 200-399 sujetos participantes $(19.1 \%)$.

- Diabetes Mellitus (7.3\%).

- Estado de reclutamiento ya completado (28\%).

Donde las multinacionales farmacéuticas fueron las principales patrocinadoras de dichos estudios clínicos, y en segundo lugar universidades, otras instituciones y laboratorios farmacéuticos colombianos (9).

Haciendo un análisis de la investigación clínica en Colombia, Desde el año 2008, cuando en Colombia se adoptó BPC para la conducción de estudios clínicos patrocinados, el promedio anual de ensayos radicados ante INVIMA ha sido de 88 , con una media de aprobación de 78 (89\%) y los no aprobados incluyen negados, desistidos y abandonados. De hecho, según las estadísticas, ocho de cada nueve estudios radicados se convierten en estudios activos, y sólo tres de cada 100 EC radicados son negados. Estos valores se han mantenido relativamente constantes a lo largo del tiempo, mostrando incluso un leve declive y ocasionales fluctuaciones. Podría concluirse que la ICP en Colombia es una actividad por desarrollar. La opinión, un tanto generalizada, de que en el país hubo una etapa dorada de ICP seguida de otra de "vacas flacas" parece que no es correcta, al menos desde 2008 en adelante (4).

Una minoría de los centros de estudios clínicos en Colombia, se encuentran afiliados con ACIC. Respetando las debidas excepciones, la falta de predictibilidad les ha quitado atractivo en el mercado mundial a la investigación clínica, y esta deficiencia se ha debido, en parte, a que su enfoque adolece de una visión empresarial rigurosa, que propenda por la excelencia en todas y cada una de sus variables. Como medida para superar las dificultades, ACIC ha propuesto a sus centros de investigación clínica la Iniciativa Nacional Para Transformar la Investigación Clínica, INTIC. El objetivo es hacer un abordaje multidisciplinario, vinculando a todos los eslabones de la cadena de la IC, para revitalizar la investigación en el país, mediante el mejoramiento de la calidad y la eficiencia de los estudios clínicos que se realizan en Colombia (10).

En cuanto a la densidad de pacientes, Colombia sigue estando por debajo de la meta esperada, países desarrollados cuentan con 70 pacientes por millón de habitantes, Argentina: 19, Chile: 11, México 6, Perú 4, Brasil 4, y Colombia tan sólo 3 por cada millón de habitantes (5). La solución para estas dificultades de investigación en Colombia está en que los centros de investigación clínica se deben unir, superar estas dificultades, si se proponen crecer en un promedio del $20 \%$ en los primeros tres años alcanzarían la meta de 110 ensayos clínicos anuales. Cinco ensayos clínicos promedio por cada centro de investigación e impulsaría a los centros de investigación a competir con calidad (5). Para subsanar las dificultades anotadas, se ha propuesto desde ACIC el manejo de indicadores de calidad los cuales han sido diseñados con un grupo de expertos en calidad de varios centros de investigación de ACIC obteniendo nueve indicadores claves que están siendo aplicados en el segundo semestre de la vigencia fiscal del 2018, los mismos son:

\section{Indicadores previos al inicio del estudio}

- Tiempo de respuesta a los estudios de factibilidad.

- Alistamiento de la información para el comité de ética (CEI).

- Tiempo para la firma de contratos .

\section{Indicadores durante la ejecución del estudio}

- Número de desviaciones críticas (muy graves) o mayores (graves).

- Tiempo para incluir el primer paciente.

- Cumplimiento del enrolamiento.

- Calidad en la impartición del consentimiento informado (CInf).

- Tasa de abandono de sujetos enrolados.

\section{Indicadores durante la finalización del estudio \\ - Captura de datos en EDC (11).}

En conclusión se puede decir que, Colombia tuvo el auge de investigación clínica con los estudios sobre osteoporosis, vacunas, mega-estudios de diabetes, hipertensión, enfermedad coronaria y en general de riesgo cardiovascular, contando con un importante crecimiento hasta el año 2015; sin embargo, desde entonces ha empezado un declive importante, explicado principalmente por la falta de una cultura de investigación, ámbito que es afectado desde el pregrado y postgrado de los profesionales de la salud, en los cuales existen muy pocas posibilidades para ahondar en el campo investigativo dentro de su malla curricular, esto se 
ve reflejado en la falta de centros de investigación clínica, de los cuales disponemos hoy en día en nuestro país y los pocos especialistas en las diferentes profesiones de la salud que incursionen en este campo, si lo comparamos con países desarrollados. Esta falta de conocimiento y cultura investigativa ha hecho que en estos momentos estemos atravesando por dificultades las cuales se ven reflejadas en la disminución de estudios clínicos aprobados y la baja densidad en cada uno de ellos, aunado al aumento en la complejidad de los nuevos estudios clínicos, donde la selección de los sujetos de investigación es cada vez más específica y con más criterios de inclusión y exclusión, lo cual dificulta incluir pacientes; por lo tanto, es importante que la cultura en investigación clínica en estudios contratados y no contratados se empiece a fomentar desde las universidades y centros de formación profesional como una necesidad para la globalización, es importante mencionar que en el momento entidades como: AVANZAR, AFIDRO, Comercio Exterior, Ministerio de Salud, INVIMA, Cámaras de Comercio y ACIC están realizando un trabajo interdisciplinario e interdependiente para lograr posicionar la investigación clínica de Colombia a los más altos estándares de calidad, de eficiencia y eficacia y generar una política en investigación clínica para Colombia. Reconocimiento especial al actual director del Invima por su gran compromiso en fortalecer los estándares de calidad y trabajar para que Colombia sea un centro de referencia internacional para estudios clínicos y desde el Invima trabajar para establecer una política pública de investigación.

Con base a lo expresado se propone a las diferentes facultades de salud del país, ASCOFAME, Colciencias y a las
EPS, a las IPS y a las asociaciones de usuarios desmitificar la investigación clínica con procedimientos de información, educación y reconocimiento al gran valor que estos procesos pueden generarle a un país, permitiendo empleos directos e indirectos, inversión de capital extranjero que mejorará la economía nacional, y generará un impacto en la salud de la población con nuevos desarrollos tecnológicos, medicamentos y poder así competir con centros de investigación clínica a nivel internacional.

\section{Referencias}

1. Molina de Salazar DI, Botero SM, Giraldo GC. Investigación clínica y ensayos clínicos ¿En qué vamos? Acta Med Colomb 2016;41:43-50.

2. Studies on Map - ClinicalTrials gov [Internet]. [citado 8 de noviembre de 2018]. Disponible en: https://clinicaltrials.gov/ct2/search/map

3. Chaves M. Situación actual de la IC en Colombia- Diagnóstico y perspectivas. septiembre de 2017;1-29.

4. Asociacion Colombiana de Centros de Investigación Clínica- Boletin Informativo ACIC. Unservicio de ACIC al fortalecimiento de la investigación Clínica. agosto de 2018;2.

5. Chaves M.Antecedentes y Perspectivas de la Investigación Clínica en ColombiaLa visión de ACIC-. 2017; ACIC.

6. Asociación Colombiana de centros de investigación Clínica. Boletín Informativo ACIC- Un servicio de ACIC al servicio al fortalecimiento de la investigación clínica en Colombia. 2 de noviembre de 2018; 2(2):1-4.

7. Salazar Molina DI, Giraldo GC. Impacto de la investigación clínica en el desarrollo de un país. Acta Med Colomb 2013; 37(4):215-9.

8. Investigación Clínica - Invima - Instituto Nacional de Vigilancia de Medicamentos y Alimentos [Internet]. [citado 1 de noviembre de 2018]. Disponible en: https:// www.invima.gov.co/buenas-Practicas-clinicas-invima

9. Jácome Roca A. Editorial: Investigación Clínica en Colombia.Academia Nacional de Medicina. junio de 2013;35(2).

10. Sanchez G. Investigación clínica en seres humanos en Colombia ¿Estamos en crisis? Acta Med Colomb 2017; 42(4):207-9.

11. Chaves M. Indicadores de calidad en la ejecución de estudios clínicos. 24 de octubre de 2017;1-7. 